\title{
Assessing multilingual lexical incorporation hypotheses through a primed picture-naming task ${ }^{*}$
}

Jorge González Alonso - University of the Basque Country

\begin{abstract}
The incorporation of new representations into the mental lexicon has raised numerous questions about the organisational principles that govern the process. A number of studies have argued that similarity between the new L3 items and existing representations in the L1 and L2 is the main incorporating force (Hall \& Ecke, 2003; Herwig, 2001). Experimental evidence obtained through a primed picture-naming task with L1 Polish-L2 English learners of L3 Russian supports Hall and Ecke's Parasitic Model of L3 vocabulary acquisition, displaying a significant main effect for both priming and proficiency. These results complement current models of vocabulary acquisition and lexical access in multilingual speakers.
\end{abstract}

Key words: third language acquisition; mental lexicon; processing; lexical access; cross-linguistic influence; Parasitic Model.

\section{INTRODUCTION}

The field of lexical representation, when considered ontogenetically, is arguably one of the most salient overlapping areas between linguistic theory, language acquisition and psycholinguistic models of processing. Any questions raised regarding the storage, organisation and access to the mental lexicon will eventually require answers that draw from our accumulated knowledge of the structure of linguistic items, the stages and pace at which they

\footnotetext{
* I am indebted to Nigel Duffield for discussion on the original study, and to María del Pilar García Mayo, Jason Rothman and two anonymous reviewers for their helpful comments on previous versions of this article. I would also like to thank Ludovica Serratrice for her editorial work. I am, of course, particularly grateful to the participants of the study.
} 
are acquired, and the interaction between the cognitive, linguistic and perceptual modules involved.

This study aims at contributing to the ever-growing body of research in this area, by focusing on the acquisition of new lexical items by multilingual speakers and the organisational principles of incorporation that guide the process. The experiment targeted L1 Polish highly advanced speakers of L2 English with Russian as their third language, at different levels of L3 attainment. Presented in this squib is the picture-naming task, in which participants were asked to name pictures in a particular language after hearing a word spoken in one of the other two. The goal of this experiment was to measure the effect of inter-linguistic connections on lexical access, and the subsequent possible interference or facilitation -also called Cross-Linguistic Influence (CLI). The task considered differences in response times (hence RTs) across two groups of proficiency (beginner and advanced) and two conditions (primed and unprimed subjects). The data obtained seem to favour the idea of a supportive role of previously acquired languages in L3 vocabulary acquisition.

\section{THEORETICAL BACKGROUND}

\subsection{Research on L3 Acquisition}

Despite an initial -and necessary- emphasis on what made the field a distinct one, and especially on the differences between acquiring a second and a third language, research on L3 acquisition has inherited some of the longest-running issues of SLA studies (see e.g. Falk \& Bardel, 2010; Rothman, Iverson \& Judy, 2011). Transfer effects are part of this group of shared concerns, and have taken many names -CLI being one of them-depending on the approach and idiosyncratic preferences of the author. Most work on both L3 syntax (e.g. Flynn, Foley \& Vinnitskaya, 2004; García Mayo, 2007; Rothman \& Cabrelli Amaro, 2010) and the multilingual lexicon (e.g. Dijkstra, 2003; Hall, 2002; Herwig, 2001) has focused either on determining the manifestation of previous linguistic knowledge influence (facilitative or non-facilitative) and its 
cognitive underpinnings or on learner strategies that work around gaps in the interlanguage with the assistance of the more established L1 and L2 systems.

Even though all of these studies tackle different research questions and thus arrive at different particular findings, there are a few conclusions that are generally accepted as shared among the L3 research community. One of the most important is that, whatever the specific mechanisms are, the active cohabitation of the various linguistic systems of a multilingual speaker influences -and often enhances- the course of further acquisition (Dijkstra, 2003). Any interference effects taking place in the multilingual mind are essentially a by-product of this coexistence, and their visibility in processing should not mislead us to the assumption that the consequences are mostly negative. There is, in fact, no point in talking about 'negative' or 'positive' transfer: this phenomenon simply happens, irrespective of whether we regard its consequences as facilitating or problematic for a foreign language learner. In any case, instances of interference are still exceptional -and, as such, visible-: while the simultaneous availability of a large number of items and structures may sometimes entail problems for selection (cf. Mahon, Costa, Peterson, Vargas \& Caramazza, 2007), the strategic support that previous linguistic systems provide for the acquisition of new languages strongly overpowers any possible inconveniences.

The study of the mental lexicon finds obvious motivations to extend research into multilingual contexts. The added complexity of whole new networks of lexical items poses a challenge for theories of representation, since any structure proposed for a lemma-concept item must take into account connections that may go beyond a single linguistic system. In particular, as it happened when bilingual mental lexicons were first assessed (see Pavlenko, 2009), the extent to which a conceptual base is shared among languages in the multilingual mind is a matter of controversy. Some authors (e.g. Hall \& Ecke, 2003; cf. Levelt, 1989) suggest that general semantic features may be external to the lexicon itself and therefore of common access for representations of different linguistic systems. 


\subsection{Lexical organisation}

Linguistic representation and the organisational principles of the lexicon are already the focus of much attention in standard lexicology, but they become a particularly puzzling subject in a multilingual scenario: to what extent are the different languages independent from each other? This question can be made from an essentially psychological perspective -what aspects of lexical representations overlap cross-linguistically?-, but also from a purely neurophysiological point of view -how much neural substrate do they share?

In 1985, Paradis published an article entitled "On the representation of two languages in one brain." Among other things, the author was concerned with the way the two languages of a bilingual co-exist, and in particular with determining whether the seemingly mutually exclusive views of a common linguistic store versus separate stores are, in fact, mutually exclusive. After considering a number of hypotheses, Paradis arrived at the conclusion that the key for differentiation lies in a functional distinction. That is: each end-state language is a subset of the neuro-functional language system.

Although Paradis (1985) never makes reference to development, Herwig (2001) provides an ontogenetic description of further language acquisition which takes the Subset Hypothesis as an end state. In Herwig's view, any new linguistic system will start as an extension of a previous one, with its elements stored in the form of variations of items within the other system: phonemes stored as allophones, morphemes as allomorphs, L2 syntactic rules as exceptional (e.g. register-driven) variations on default syntax, etc. - the scenario that Paradis (1985: 20) calls the Extended System Hypothesis. Recurrent use of the new items would eventually fix them as a separate, functionally distinct network within the larger system ${ }^{1}$.

\footnotetext{
${ }^{1}$ This recurrent use is understood in terms of activation. Interactive Activation models, such as McClelland and Rumelhart's (1981), view linguistic representations as nodes in a network which compete for selection. Upon triggering, a node will spread this activation through excitatory links to its related nodes of the subset and the superset levels, while sending inhibition signals to competing nodes. After a number of cycles, the most highly activated item is selected.
} 
A number of predictions may be derived from this idea. One of the most important is that novice L3 learners are likely to be much more prone to showing priming and interference effects, due to the weak functional segregation of their linguistic systems. Cross-linguistic influence may then show at all levels, from phonology -which concerns us most here- to syntax. The status of L3 representations as variations of either L1 or L2 items would complicate inhibition and therefore favour interference.

\subsection{Lexical incorporation and the Parasitic Model}

Hall and Ecke's (2003) Parasitic Model is an intuitive idea that relates to theories of associative memory and, more within the field of lexical organisation, to Herwig's account of plurilingual lexical organisation. The main contention of the model is that new representations in the third language are incorporated into the speaker's linguistic network through 'points of contact' with existing representations in the L1 and the L2 -i.e. in the authors' terminology, new L3 entries are parasitic upon well-established L1 and L2 hosts ${ }^{2}$. These common features serve as a cue for retrieval of the new items, which are therefore dependent on the initial crosslinguistic links until they receive sufficient activation to become strong, independent representations.

For the authors, there are three distinguishable types of cross-linguistic influence (CLI): Acquisition CLI (ACLI), Performance CLI and Competence CLI. The first would be the mechanism by which a multilingual speaker makes use of pre-existing knowledge to build a vocabulary for the new language based on similarities at any level of representation with the existing linguistic systems at hand. This idea is close to Herwig's (2001) model: the associative links established between the new L3 representations and existing L1 and L2 items enhance the creation of a dependent network that will eventually acquire its own status of separate linguistic system as proficiency increases. Until this happens, frequent errors are likely to take place in the

\footnotetext{
${ }^{2}$ The model also contemplates the possibility of the host being a well-established L3 representation. Rhyme, for example, may be a frequent form-based point of similarity exploited in these cases.
} 
speaker's production which are attributable to a variant of what Hall and Ecke call 'competence CLI': in search for the relevant L3 item, if this is underspecified speakers might instead produce the L1/L2/L3 representation that serves as a host or cue of the target, even if this is not a translation equivalent. This is perhaps most common in cases where both representations share a word-class -e.g. when they are two verbs with analogous argument structures.

When looking at how specifically parasitic associations take place, it is important to consider the process whereby new items are retrieved in speech production. Upon activation of certain conceptual features, these will in turn select an appropriate lexical entry in the target language. Assuming that a large part of the conceptual repertoire of a multilingual speaker is common to all linguistic systems, the question is how the activation of a particular concept may lead to the selection of a weak or under-represented word form, instead of a non-target language item or a situation of non-articulation due to unsuccessful retrieval.

To describe the levels at which cross-linguistic connections are established, we must first assume a basic structure of lexical entries. The Parasitic Model uses the concept of lexical triads, very much in line with a traditional approach associating concept, morpho-phonological form (also called lexeme, e.g. in Levelt, 1989) and syntactic specification. Hall and Ecke speak of concept, form and frame, respectively, with the last two being more tightly related (see figure 1). Although the connection to the 'meaning' dimension has been considered an intra-linguistic phenomenon by some authors, conceptual features are assumed here to be external to the lexicon itself (Hall \& Ecke, 2003: 75). In the model under review, all examples of CLI produce associations within the form-frame dominion, although analogous or equivalent conceptual representations are the most common trigger for these connections.

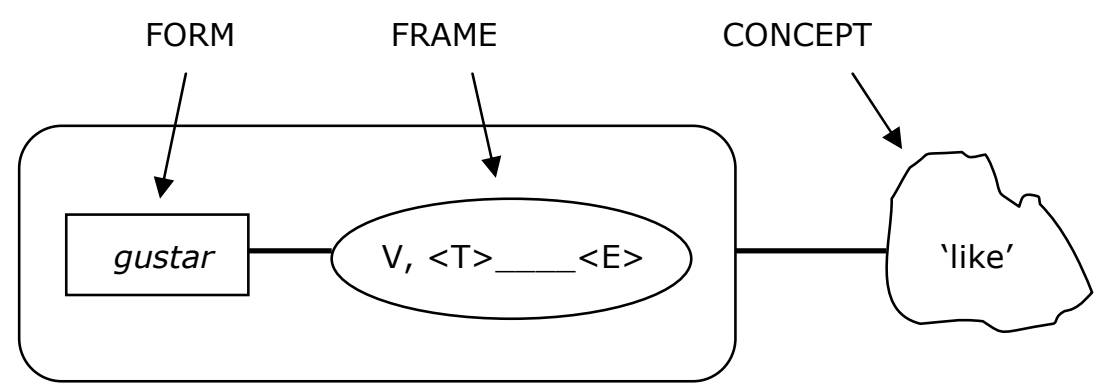


Figure 1. Lexical triad for the Spanish word gustar 'like' (adapted from Hall \& Ecke,

It is important to note here that the idiosyncrasy of parasitic relations presents a problem for a priori assumptions. Predictions are hard to make: the kind of associations that work for a particular speaker might not take place in the mind of another. That is: we can hypothesise, on the basis of formal or structural similarity, that an L3 item might be more easily remembered if incorporated through a particular L1 or L2 representation, but this is no guarantee that the acquisition mechanisms of every speaker are going to make use of that potential. Although the model had previously been tested using experimental methods (Hall, 2002), the evidence provided in Hall and Ecke (2003) was obtained through the recording, transcription and analysis of five-minute interviews, thus working around the problem of unpredictability. In this sense, experiments like the one reported in the present study depend more on an appropriate choice of materials. Nevertheless, the type of ACLI assumed here (phonology-based) is slightly safer than other kinds of predictions, e.g. those based on syntactic frame or semantic associations: phonological resemblance is perceptually more salient than other potential similarities, and it does not rely on any further specification of the lexical entry; this leaves open the possibility of incorporation of those new items from which the learner has only fragmentary semantic, syntactic or pragmatic information.

However, although form resemblance may be the first criterion for incorporation given a particular new word form, the model assumes that connections will also be established between the new item and its perceived translation equivalents, independently of form resemblance. Thus, an L3 word incorporated through form similarity with a semantically unrelated L1 lexical item will be connected, at least through the initial stages of lexical specification, to two different forms within the L1: its phonologically similar host and its translation equivalent -plus its L2 translation equivalent. This situation becomes particularly complex in the case of cognates, both false (faux amis) and true. The next section discusses some of the findings reported in the 
literature concerning the effects of phonological similarity, as well as the special case of cognates within the Parasitic Model.

\subsection{Effects of cross-linguistic form similarity on lexical access}

From the many factors influencing CLI listed by Hall \& Ecke (2003; see the detailed table on page 73), the one chosen for the experiment reported here was the degree of form similarity between items. The fact that the language combination was Polish-English-Russian, however, provided an interesting dissociation of two aspects which are often inextricably bound within the form domain: orthography and phonology. Given the radical differences -and the misleading apparent parallels- between the Cyrillic and Latin alphabets, 'form similarity' in this case can only refer to phonology, since the identification takes place between items from two languages with a Latin script (Polish or English) and items from a language spelled with the Cyrillic alphabet (Russian).

The literature assigns different effects to phonological and orthographic overlap, although the results seem to vary depending on whether the studies focus on comprehension or production; there also seems to be an influence of the experimental paradigm used and the modality of the stimuli presented -auditory or visual. In a series of experiments with L1 DutchL2 English speakers including a progressive demasking task and a visual lexical decision task, Dijkstra, Grainger and Van Heuven (1999) report inhibitory effects of phonological overlap, while semantic and orthographic similarity are regarded as facilitatory in lexical retrieval. To accommodate both these results and previous findings by Dijkstra, Van Jaarsveld and Ten Brinke (1998) which highlight the importance of word frequency, the authors propose a new version of the Bilingual Interactive Activation (BIA) model in which interlingual homographs are represented once for each language.

Studies conducted within the picture-word interference (PWI) paradigm, on the other hand, have generally found facilitatory effects of cross-linguistic phonological similarity. Costa, Miozzo and Caramazza (1999) tested Catalan-Spanish bilinguals in a series of picture-word 
interference experiments. Their results show facilitation when there is phonological overlap between the distractor and the target word, even if the former belongs to the non-target language. The authors attribute this effect to sub-lexical processes of interaction between graphemes and phonemes, since the translation equivalents of phonologically similar words did not seem to enhance faster naming latencies.

Also within the PWI paradigm, Hermans, Bongaerts, De Bot and Schreuder (1998) conducted two experiments in which they manipulated the stimulus-onset asynchronies (SOAs) of the picture (to be named in English) and the distractor. These could be an English phonological neighbour (experiment 1), a phonologically related Dutch word (experiment 2) or an English word which was phonologically related to the Dutch translation of the target (what they call the Phono-Dutch condition, present in both experiments). Similarly to Costa et al. (1999), the authors found a facilitatory effect of phonological neighbours at all SOAs (-300, 150, 0 and +150 milliseconds). They did not, however, find facilitation of phonologically similar Dutch words except at $+150 \mathrm{~ms}$. Interestingly, they report interference effects in the Phono-Dutch condition at all SOAs except $+150 \mathrm{~ms}$, which somehow contradicts the results in Costa et al. (1999) as regards the activation of translation equivalents. It may be the case, of course, that the difference in the presentation of distractors (visual vs. auditory) plays a role in the size of the effect observed.

Cognates, which could be defined -simplifying slightly- as etymologically related lexical items from two different languages which share both formal and conceptual features, make a particularly complex group. Hall (2002) presented Spanish speaking learners of English as a foreign language (EFL) with non-words that were either pseudocognates -sharing at least twothirds of form- or noncognates of existing Spanish words. Participants almost invariably reported more familiarity with pseudocognates, for which they hypothesised a meaning that was very frequently constrained by the conceptual features of the non-word's perceived Spanish cognate. This, as the author explains, suggests that learners make the reasonable assumption following natural principles of economy- that shared form implies shared meaning (Hall, 2002: 
81). It also suggests that assuming cognate status for any pair of relatively analogous words helps the learner anchor the new form to an established frame and concept, providing a provisional but highly specified representation. The divergence in meaning should not produce any kind of interference until it is noticed by the speaker, and even then the parasitic link between the two word forms is likely to remain strong, by force of repeated activation during the initial stages.

\section{RESEARCH QUESTIONS AND HYPOTHESES}

Assuming that lexical access reflects lexical organisation, the associations described above should be visible in real-time processing, the activation of the host entailing automatic activation of the parasite. In a primed picture-naming task, this would translate into the priming of the host enhancing the subsequent retrieval of the parasite. Should we expect differences, however, between novice and advanced learners, with the former group displaying a larger effect of priming? My predictions were the following:

1. Priming participants with the host L1 or L2 representation of a target word will result in shorter naming latencies in comparison with target words preceded by unrelated L1 or L2 lexical items.

2. Novice L3 learners will show a greater effect of priming than their advanced peers, with the difference in response latencies between primed and unprimed participants being greater in the low proficiency group.

\section{THE STUDY}

The evidence supplied by Hall and Ecke (2003) in support of their proposal was obtained through the analysis of relatively spontaneous production data (interviews), in which relevant patterns in line with the model's predictions were discovered. It seemed interesting to assess the 
predictive power of the model through a methodology that assumed certain cross-linguistic connections a priori, in line with Hall (2002). In order to do this, only one of the many possible points of contact identified by Hall and Ecke (2003) was used: form resemblance.

\subsection{Materials}

Questionnaire

All participants filled in a questionnaire that had the main purpose of assisting their classification into different proficiency groups. It was divided into three sections:

1) Linguistic background: gathering information about the speaker's relationship with all three languages used in the experiment, and the status conceded to each of them. Questions addressed, among other aspects, their mother tongue, their explicit learning experiences and their contact with a naturalistic language environment.

2) Self-assessment and vocabulary, in which participants were first asked to rate themselves in the third language using a Likert-type 7-point scale (Likert, 1932), and then translated a number of words between the relevant languages, mainly to ensure that they already knew the Russian target items contained in the picture-naming task.

3) Counting task: largely based on Dornic's (1980) considerations about the importance of both perceived effort and objective measurement in switching costs, when assessing the (a)symmetry between the two languages of a bilingual. The main idea here was to ensure that Polish and English were sufficiently balanced. Participants were asked to count down from 21 to 1 , starting in English (L2) and switching between Polish (L1) and English at every number.

\section{Word pairs and pictures}

In order to reveal potential parasitic links in the lexical organisation of the participants' linguistic systems, 16 word pairs were used that combined Russian target words with Polish (10 pairs) and English (6 pairs) potential hosts (see table 1). False cognates provided a practical way of finding stimuli with an appropriate degree of phonological overlap, which was subsequently 
confirmed by two native speakers of each of the languages involved. This, in turn, introduced a derived variable, since the literature typically identifies two types of false cognates: true false cognates and indirect cognates ${ }^{3}$. To the first group belong word-pairs with a purely coincidental form resemblance; to the second, word-pairs whose degree of form similarity is rooted in a common etymology or in interlanguage borrowing, despite a posterior semantic drift that left the two words sharing only a number of semantic features -which disqualifies them as translation equivalents. Although the number of tokens belonging to each group was not controlled for in the design, post-hoc analyses can be run on the response-time data, since the relevant word-pairs can be assigned to one or the other category easily (see the discussion section).

\begin{tabular}{|c|c|c|}
\hline POLISH & ENGLISH & RUSSIAN \\
\hline głaz (rock) & & глаз (еуе) \\
\hline dworzec (train station) & & дворец (palace) \\
\hline dywan (carpet) & & диван (sofa) \\
\hline kawior (caviar) & & ковёр (carpet) \\
\hline mecz (match) & & меч (sword) \\
\hline niedziela (Sunday) & & неделя (week) \\
\hline pamiętnik (diary) & & памятник (monument) \\
\hline uroda (beauty) & & урод (freak) \\
\hline młodzieniec (young man) & & младенец (baby) \\
\hline \multirow[t]{7}{*}{ płot (fence) } & & плот (raft) \\
\hline & clay & клей (glue) \\
\hline & mark & марка (stamp) \\
\hline & more & mope (sea) \\
\hline & fabric & фабрика (factory) \\
\hline & auditorium & аудитория (audience) \\
\hline & cell & цель (target) \\
\hline
\end{tabular}

\footnotetext{
${ }^{3}$ See Hall (2002) for a brief overview of the literature on degree of cognateness.
} 
Table 1. List of the 16 relevant word pairs used in the primed condition. Target items were always Russian, whereas the hypothesised hosts were Polish (10) and English (6) words

The word list was completed with another 120 word pairs which were used as fillers and distractors. It contained the following combinations: 20 English-Russian, 20 Polish-Russian, 20 Russian-English, 20 Russian-Polish, 20 English-Polish and 20 Polish-English pairs. These words had no connections with each other: their semantics, phonology and argument structure did not coincide -in fact in most cases the word class was different. To make the picture-naming task as unambiguous as possible, the 136 images selected were clear colour photographs showing the object or entity that should be named. They all stood against a white background which made the object the only perceivable item on screen.

\subsection{Participants and procedure}

Given the inherent difficulty of finding speakers that met the desired profile (highly proficient L1 Polish- L2 English speakers L3 learners of Russian), a total of 12 participants (all students or research staff at the University of Sheffield) were tested. The final population consisted of 4 females and 8 males. Ages ranged from 19 to 64, although the mean age was 28 with a median of 23. All participants had Polish as their mother tongue and spoke English as a fluent L2, with the exception of two people who were early bilinguals. Russian was spoken as a third language, with different levels of proficiency.

The size of the sample made it impractical to assess the participant's proficiency in the third language through external measures (e.g. certified placement tests). Instead, they were assigned to a proficiency group or the other based on data from the linguistic background section of the questionnaire. The notable difference between a novice and an advanced foreign language learner, however, left little margin of error when judging by these parameters: beginners had received little instruction in Russian (one or two academic years), while advanced participants were all students of Russian language and linguistics at university level. 
After their classification into proficiency groups, participants were randomly assigned to a 'primed' or an 'unprimed' sub-group. In total, 9 participants were classified as beginners (5 primed, 4 unprimed), and 3 as advanced (1 primed, 2 unprimed). The group distribution was partly influenced by a further task taken by the more advanced participants, since the experiment reported here is one of two pertaining to a larger project. Given space limitations, only the picture-naming task is discussed in detail, although the findings across the two experiments largely correlate.

\section{Procedure}

The picture-naming task was programmed into a DmDX file that presented the stimuli and controlled the synchronisation of pictures, words and sound files. The programme measured the response times of participants and recorded their answers through the built-in plug-ins DigitalVOX and RecordVocal, respectively. Before proceeding to the actual task file, participants were played a rehearsal of 10 items (made up of 3 frames each), with which they could become familiar with the procedure. The frames of both the rehearsal and task files (for both the primed and the unprimed condition) followed the sequence depicted in figure 2 .

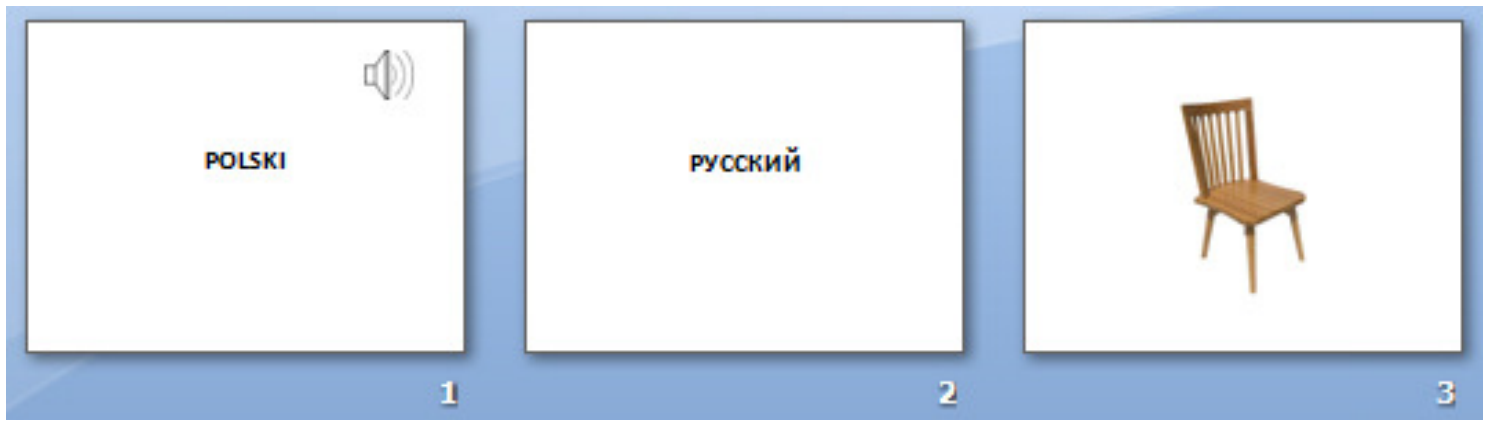

Figure 2. Sequence of frames in the items of the DmDX task

In (1), the participant is presented with the name of one of the three languages involved, spelled in that language, in capital letters, i.e. "ENGLISH", "POLSKI" or "РУССКИЙ". Тhis visual display is accompanied by a sound recording of a word pronounced in the language 
named by the text -the prime or potential host in the case of the primed condition, and an unrelated word in the case of the unprimed condition. The presence of the language's name is also meant to filter out any activation of the target $\mathrm{L} 3$ representation during the priming frame. Frame (1) is displayed for as long as the recording plays, which averages at 0.9 seconds for all items. Immediately after, the screen changes -with no transition clearing- to present frame (2), with the name of a different language from the first. This is a cue for the participant: whatever language is written in frame (2) of every item, that is the one s/he should use to name the picture in frame (3). This appears exactly after 500 milliseconds, and displays one of the pictures described above. The screen is cleared after a time-out period of 3.5 seconds, and the next item starts. There is virtually no delay between items $(119 \mathrm{~ms})$. The items of the rehearsal and task files for both the primed and the unprimed condition are actually identical sequence-wise; it is only the relation between the word participants hear and the name of the picture that changes in the primed condition, the Russian target words are paired up with their potential hosts, whereas in the unprimed condition they are paired up with unrelated Polish or English words.

Costa et al. (1999) contend that, once a response language is identified and stably activated, competition among lexical items is very much restricted to those belonging to the relevant linguistic system. To avoid this effect, the three languages used in this experiment were randomly combined and displayed, preventing participants from identifying a constant response-relevant language.

\section{RESULTS}

The task had an unconventional design, in that comparisons were based not on response time (RT) differences between target and filler items, but rather between primed (all target items primed) and unprimed (no primed target items) subjects. In this sense, there were only two broad groups of items: target items and fillers, with the former subdivided into two categories according to the language to which the host belonged. Thus, there were two between-subjects 
independent variables, priming (primed vs. unprimed) and proficiency (high vs. low); and one between-items independent variable, language of the prime (Polish vs. English), configuring a $2 \times 2 \times 2$ design. Table 2 contains the mean response times and standard deviations for every group of subjects.

\begin{tabular}{|c|c|c|c|c|}
\hline & \multicolumn{2}{|c|}{ High proficiency } & \multicolumn{2}{|c|}{ Low proficiency } \\
\hline & Primed & Unprimed & Primed & Unprimed \\
\hline Mean RT & 1208.85 & 1396.49 & 1486.95 & 1828.94 \\
\hline $\begin{array}{l}\text { Standard } \\
\text { deviation }\end{array}$ & - & 147.07 & 216.11 & 85.79 \\
\hline Totals (prof.) & \multicolumn{2}{|c|}{$1256.66(S D=165.66)$} & \multicolumn{2}{|c|}{$1586.33(S D=237.61)$} \\
\hline Totals (priming) & \multicolumn{2}{|c|}{ Primed: 1383.77 (SD = 239.07) } & \multicolumn{2}{|c|}{ Unprimed: $1624.05(S D=241.7)$} \\
\hline
\end{tabular}

Table 2. Mean RTs (in milliseconds) for each group of proficiency x priming, and general mean RTs for proficiency and priming

A first look at the means of each group seems to indicate that both priming and high proficiency produce faster latencies $(240.28 \mathrm{~ms}$ difference between primed and unprimed subjects; $329.67 \mathrm{~ms}$ for high vs. low proficiency). This was confirmed by paired-samples t-tests performed between the groups, in which significance was obtained in every case: primed low proficiency vs. unprimed low proficiency $(\mathrm{t}(15)=-3.26, \mathrm{p}<0.01)$; primed high proficiency vs. unprimed high proficiency $(\mathrm{t}(15)=-2.59, \mathrm{p}=0.021)$; primed high proficiency vs. primed low proficiency $(\mathrm{t}(15)=-5.21, \mathrm{p}<0.01)$; unprimed high proficiency vs. unprimed low proficiency $(\mathrm{t}(15)=-5.02, \mathrm{p}<0.01)$

A repeated measures ANOVA was performed on the by-items data, displaying significant main effects for both priming $(\mathrm{F}(1,14)=11.88, \mathrm{p}<0.01)$ and proficiency $(\mathrm{F}(1,14)=35.55, \mathrm{p}<$ $0.01)$, but not for an interaction of both factors $(F(1,14)=1.66, p=0.22)$. The language of the prime did not have any significant effect in combination with these factors: priming $\mathrm{x}$ language 
$(F(1,14)=0.19, p=0.67)$, proficiency $x$ language $(F(1,14)=0.3, p=0.59)$ and priming $x$ proficiency x language $(\mathrm{F}(1,14)=0.63, \mathrm{p}=0.44)$ all show $p$ values which are very far from significance. Analyses of the data arranged by subjects were not performed, due to the small size of the sample and the distribution of participants.

\section{DISCUSSION}

The results of the picture-naming task support the Parasitic Model proposed by Hall and Ecke and point in the direction of the initial predictions: priming target L3 items with their hypothesised host L1 or L2 representations enhanced the retrieval of these words, in spite of their not being translation equivalents. This effect was, as expected, more noticeable in low proficiency speakers, for whom lexical selection in the third language is generally slower, perhaps as a result of under-specification and incomplete representation. The significance of the priming effect was notably higher in that group $(\mathrm{p}<0.01$ vs. $\mathrm{p}=0.02)$.

There are many possible explanations for this difference, which is in fact likely to result from the combination of a number of factors. The most important is perhaps the speed and accuracy that learners achieve as they gain fluency in the new language. Advanced learners are already relatively fast at naming pictures, and so the impact of priming in their response latencies will be smaller. A secondary explanation is that the interplay of lexical and conceptual connections derived from parasitic associations may begin to produce some interference past the initial stages. While for novice learners lexical items incorporated through phonological similarity are frequently just extra word forms attached to certain lexical triads, the new entries have been gradually specified in the lexicon of an advanced speaker, establishing connections with their translation equivalents. This entails higher and more diverse conceptual activation, and perhaps also slightly slower processing.

In line with this possible semantic interference was the potential difference between the two categories of stimuli (as regards cognate status): true false cognates and indirect cognates. 
Some authors (e.g. Odlin, 1989; Ringbom, 1987) have pointed at a hindering effect of false cognates in lexical retrieval. Making a more fine-grained distinction, it could be argued that indirect cognates, by force of having shared semantic features, may display facilitatory or inhibitory effects, compared to the more semantically neutral category of true false cognates. However, post-hoc analyses do not seem to find a significant difference between the mean RTs for the two groups: an independent-samples t-test returned a $p$ value well within chance levels $(\mathrm{t}(5)=-0.37, \mathrm{p}=0.71)$

\subsection{Early L3 acquisition: parasitic links as mnemonic cues}

The fact that the picture-naming task revealed a strong effect of priming suggests a critical relevance of parasitic links in lexical selection. These are created upon incorporation of a new L3 representation, making use of any points of contact this may have with existing L1, L2 or L3 items: similarities in phonology, semantic field, syntax, morphology, pragmatics, etc. A crosslinguistic excitatory connection is thus established between the two representations -and, eventually, between the new item and its translation equivalents-, spreading activation beyond language barriers. We can only presume that this process, given its flexible nature, is a generalised (or "default", as Hall and Ecke (2003) have called it) mechanism of vocabulary acquisition. However, determining at what level of representation the connection is created is a particularly difficult task, especially since associations are likely to take place at more than one level.

At these first stages, the L3 network is established and begins creating increasingly strong intra-linguistic links among its elements, which in most cases are sustained -despite their underspecification- by their association to nodes of the L1 and L2 networks. Parasitic links are explored in service of a faster retrieval of L3 representations, whose regular activation provides them with some saliency and facilitates the creation of a progressively detailed structure. Eventually, a relative independence from their hosts is achieved: the level of activation from conceptual nodes increases and becomes stronger than that received through the parasitic link, 
which is essentially lexical in nature. This phase would correspond to an intermediate level of proficiency, which has not been tested in this study.

\subsection{Advanced stages: emancipation and inhibition}

As the L3 network is developed, the new language becomes more defined. Following Dijkstra's (2003) Multilingual Interactive Activation Model (MIA, an extension of the Bilingual Interactive Activation Model), this results in the development of a progressively stronger L3 language node which feeds a higher level of inhibition towards members of the first and second languages. In the context of parasitic relations, the activation of the host -which frequently belongs to the $\mathrm{L} 1$ or the $\mathrm{L} 2-$ is reduced as soon as the $\mathrm{L} 3$ is identified as the response language, making its contribution to the selection of the target L3 representation a modest one. We may assume that, over time, the lack of joint activation undermines the associative bond. As members of competing linguistic systems, the need for differentiation transforms the nature of the parasitic link, which now carries mostly inhibitory signals that support the general pattern of inhibition/activation among and within the language networks of the multilingual lexicon.

Further research in this line will face the problem of obscurity in advanced stages of proficiency, when learners' behaviour comes deceptively close to native-like performance, but its findings will be particularly useful to help us better understand the developmental patterns of multilingual lexicons. 


\section{REFERENCES}

Costa, A., Miozzo, M., \& Caramazza, A. (1999). Lexical selection in bilinguals: Do words in the bilingual's two lexicons compete for selection? Journal of Memory and Language, 41, 365-397.

Dijkstra, T. (2003). Lexical processing in bilinguals and multilinguals: The word selection problem. In Cenoz, J., Hufeisen, B. \& Jessner, U., (Eds.), The multilingual lexicon (pp. 11-26). Norwell, MA: Kluwer.

Dijkstra, T., Grainger, J. \& Van Heuven, W. J. B. (1999). Recognition of cognates and interlingual homographs: The neglected role of phonology. Journal of Memory and Language, 41, 496-518.

Dijkstra, A., Van Jaarsveld, H., \& Ten Brinke, S. (1998). Interlingual homograph recognition: Effects of task demands and language intermixing. Bilingualism: Language and Cognition, 1(1), 51-66.

Dornic, S. (1980). Language dominance, spare capacity and perceived effort in bilinguals. Ergonomics, 23(4), 369-377.

Falk, Y. \& Bardel, C. (2010). The study of the role of the background languages in third language acquisition. The state of the art. International Review of Applied Linguistics, IRAL, 45, 185-219.

Flynn, S., Foley, C. \& Vinnitskaya, I. (2004). The cumulative-enhancement model for language acquisition: comparing adults' and children's patterns of development in first, second and third language acquisition of relative clauses. The International Journal of Multilingualism, 1, 3-16.

García Mayo, M. P. (2007). Universal Grammar and the acquisition of L3 syntax. Vigo International Journal of Applied Linguistics, 4, 25-40.

Hall, C. (2002). The automatic cognate form assumption: Evidence for the Parasitic Model of vocabulary development. International Review of Applied Linguistics, IRAL, 40(2), 69-87.

Hall, C. \& Ecke, P. (2003). Parasitism as a default mechanism in L3 vocabulary acquisition. In Cenoz, J., Hufeisen, B. \& Jessner, U. (Eds.), The multilingual lexicon (pp. 71-85). Norwell, MA: Kluwer.

Hermans, D., Bongaerts, T., De Bot, K. \& Schreuder, R. (1998). Producing words in a foreign language: Can speakers prevent interference from their first language? Bilingualism: Language and Cognition, $1(3), 213-229$.

Herwig, A. (2001). Plurilingual lexical organisation: Evidence from lexical processing in L1-L2-L3-L4 translation. In Cenoz, J., Hufeisen, B. \& Jessner, U. (Eds.), Cross-linguistic influence in third language acquisition: Psycholinguistic perspectives (pp. 115-137). Buffalo: Multilingual Matters. Series 'Bilingual education and bilingualism', edited by Hornberger and Baker, vol. 13.

Levelt, W. J. M. (1989). Speaking: From intention to articulation. Cambridge, MA: MIT Press.

Likert, R. (1932). A technique for the measurement of attitudes. Archives of Psychology, 140, 1-55. 
Mahon, B.Z., Costa, A., Peterson, R., Vargas, K.A., \& Caramazza, A. (2007). Lexical selection is not by competition: A reinterpretation of semantic interference and facilitation effects in the picture-word interference paradigm. Journal of Experimental Psychology: Learning, Memory and Cognition, 33, 503-535.

McClelland, J.L. \& Rumelhart, D.E. (1981). An interactive activation model of context effects in letter perception. Part 1: An account of basic findings. Psychological Review, 88(5), 375-405.

Odlin, Terence (1989). Language transfer: Cross-linguistic influence in language learning. Cambridge: Cambridge University Press.

Paradis, M. (1985). On the representation of two languages in one brain. Language Sciences, 7(1), 1-39.

Pavlenko, A. (2009). Preface: Time for new metaphors? In Pavlenko, A. (Ed.), The bilingual mental lexicon: Interdisciplinary approaches (pp. xi-xv). Buffalo: Multilingual Matters. Series 'Bilingual education and bilingualism', edited by Hornberger and Baker.

Ringbom, H. (1987). The role of the first language in foreign language learning. Clevedon, PH: Multilingual Matters.

Rothman, J. \& Cabrelli Amaro, J. (2010). What variables condition syntactic transfer? A look at the L3 initial state. Second Language Research, 26(2), 189-218.

Rothman, J., Iverson, M., \& Judy, T. (2011). Some notes on the generative study of L3 acquisition. Second Language Research, 27(1), 5-19. 